\title{
Cauda equina syndrome secondary to lumbar disc herniation. Report of three cases
}

\author{
Danil Adam ${ }^{1,2}$, Ioana Hornea ${ }^{2}$ \\ ${ }^{1}$ Associate Professor, Department of Neurosurgery, "Carol Davila” University of \\ Medicine and Pharmacy, Bucharest, Romania \\ ${ }^{2}$ Emergency Clinical "St. Pantelimon" Hospital, Șos. Pantelimon 240, Sect.2, \\ Bucharest, Romania \\ Corresponding author: Danil Adam, e-mail: adam_danil@yahoo.com
}

\begin{abstract}
Cauda equina syndrome produced by the herniated lumbar intervertebral disc is a rare disorder that, if is undiagnosed and untreated in time, can have serious consequences for the patient and medicolegal implications for the surgeon.

We report the clinical evolution of 3 patients with lumbar spinal stenosis who still present sphincterian and sexual dysfunctions many years after surgery, even if they were operated on immediately after admission.

Reviewing the literature on this subject has allowed us to emphasize symptomes, pathophysiological mechanism and management regarding patients with lumbar spinal stenosis.

The surgical decompression is indicated as soon as possible.

The surgical decompression within 48 hours from onset allows maximum improvement of symptoms and absolves the surgeon of any medico-legal liability.
\end{abstract}

Key words: cauda equina syndrome, lumbar herniated disc.

\section{Introduction}

Cauda equina syndrome (CES) is a rare but devastating neurologic condition resulting from many causes, most commonly secondary to a massive prolapsed intervertebral disc.

CES secondary to intervertebral disc herniation was first reported in 1929, by Dandy (1). CES may arise also from neoplastic, traumatic, inflammatory causes. The mechanism of injury to the nerves in CES is due to mechanical pressure, ischemia or venous congestion (2).

Patients complaines are bilateral leg pain and weakness saddle anesthesia and urinary or rectal incontinence. Urgent surgery is required to halt or reverse neurological signs and symptoms (3). Without surgery CES progresses and became permanent (4, $5)$. With surgery, recovery occurs over years.

It is generally accepted that surgical decompression within 48 hours of the onset of symptoms is necessary for maximum improvement of clinical signs and symptoms (6).

We report our 3 cases with CES followed between 1 to 4 years postoperatively and review the literature concerning factors that influence the outcome. 


\section{Clinical presentation}

\section{Case 1}

A 39-year-old male was operated for lumbar disc herniation at L3-L4 level, 3 years before.

The CT-scan revealed disc protrusions at L4-L5 and L5-S1 level, with 60\% lumbar spinal stenosis (Figure 1).

He continued to do heavy works as unqualified worker and returned with left leg pain, saddle paresthesia which began 5 days prior and urinary incontinence since 1 day.

Neurologic examination revealed a healthy man in good physical condition.

The range of motion of the lumbar spine was painfully reduced in all directions, with anterior flexion limited at 10 degrees. Bilateral straight leg raising was painfully limited at 40 degrees. The left achilles tendon reflex was absent.

All other deep tendon reflexes were normal. Sensation to light touch and pin prick was decreased in L5 and S1 dermatomes.

A CT-scan of the lumbar spine revealed a large median disc herniation at the L4-L5 and L5-S1 levels.

The patient underwent a discectomy at both levels through bilateral interlamar approach.

Sexual dysfunction and bowel problems persisted at 1 year follow-up making the patient very unhappy. He tried to commit suicide. At 4 year follow-up sexual dysfunction is still present, with very difficult erection and ejaculation even with ENTRAN taken before sexual intercourse.

\section{Case 2}

A 52-years-old man presented in emergency room complaining of back and leg pain on left side since 1 week and paresthesias in left buttock and genitals since 1 day.

Neurologic examination revealed a limitation of lumbar spine motions with anterior flexion at 45 degrees an left straight leg raising test limited at 45 degrees. Patelar and achilles reflexes were present. Muscle strength testing demonstrated weak left dorsal extensions of left halux, graded 3/5. Sensation to touch and pin prick was decreased along the left L5 dermatome.

MRI of lumbar spine revealed a large L4-L5 disc herniation, with $12 \mathrm{~mm}$ anteroposterior diameter (Figure 2).

The patient underwent an urgent intervention the next day. During intervention for discectomy, a dural tear appeared without consequences.

In the postoperative period saddle anesthesia persisted. At 1 month follow-up he had constipation. A glycerine suppository was inefficient, and enema was necessary for clearing the rectum.

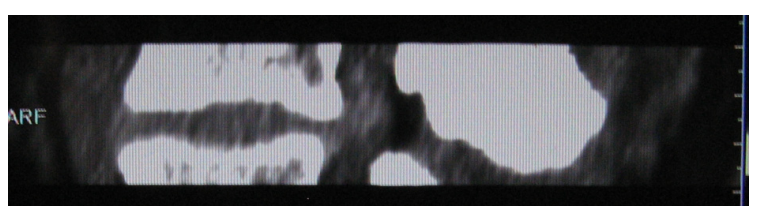

Figure 1

CT-scan of lumbar spine, sagital section at L4-L5 level shows a huge herniated disc

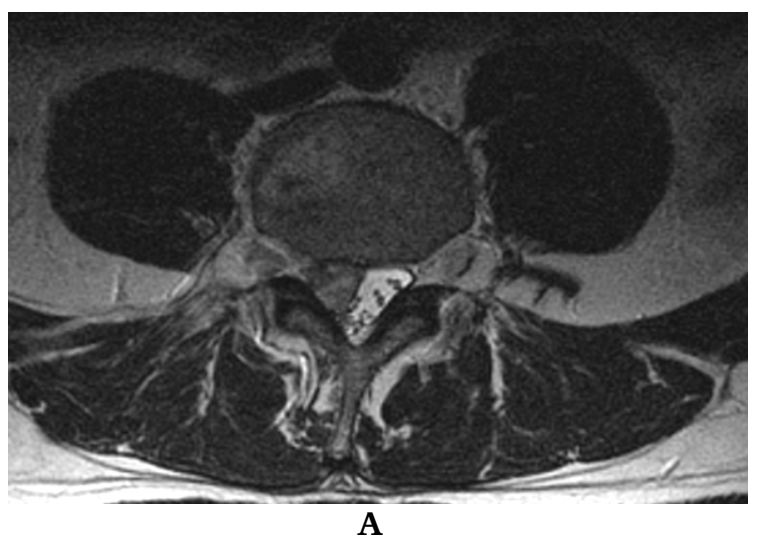


DOI: 10.2478/romneu-2013-0009

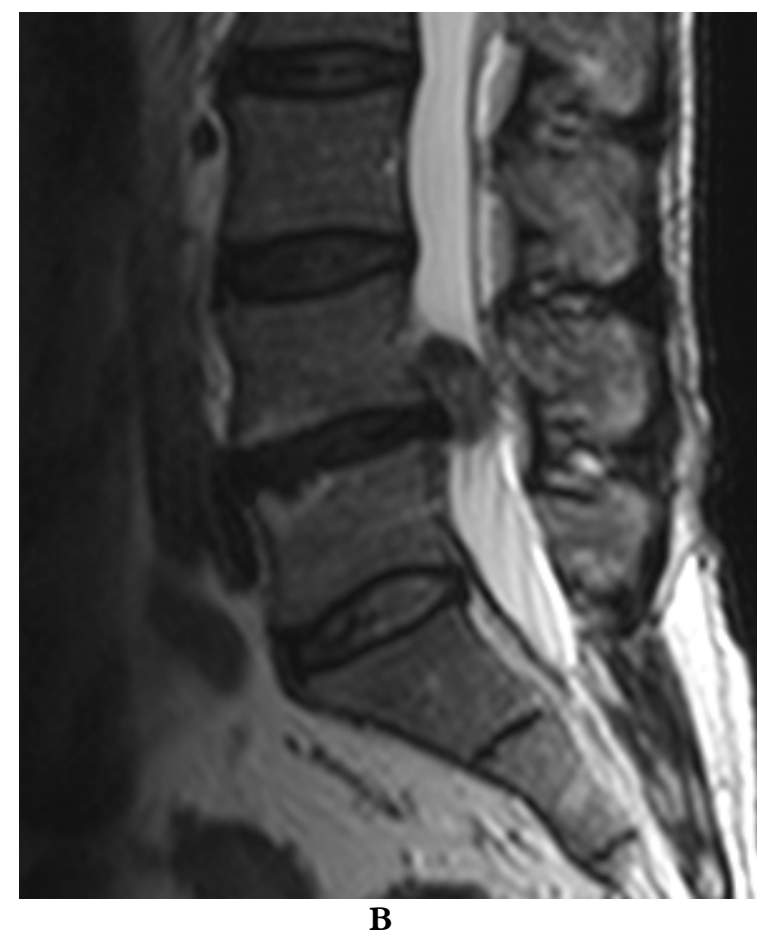

Figure 2

Preoperative MRI: axial section (A) and sagital section (B) reveal a large L4-L5 disc herniation

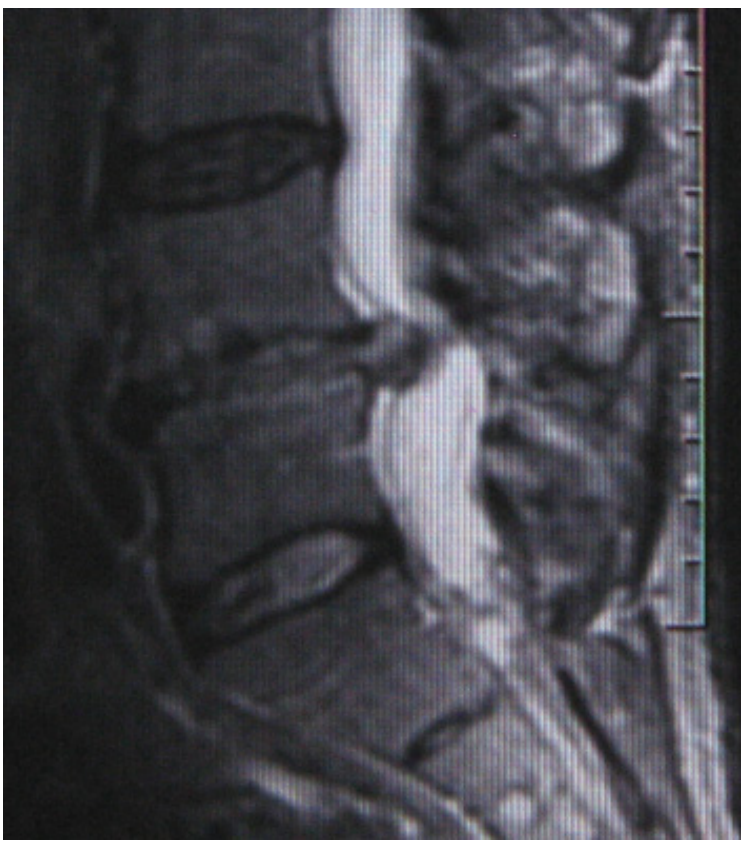

Figure 3

T2-weighted MRI of lumbar spine, sagital section, demonstrates a large L4-L5 disc herniation

\section{Case 3}

A 41-years-old female, complaining of bilateral sciatica and perigenital and perianal paresthesias since three days, presented at emergency room.

Neurologic examination revealed a reduced range of motion of the lumbar spine, with anterior flexion limited at 45 degrees and straight leg raising test at 45 degrees. She had achilles tendon reflexes bilateral abolished, SPI paresis on the left side, and urinary retention appeared in the day of admission.

Lumbar CT and MRI scans demonstrated a large central disc herniation at L4-L5 level, $11 \mathrm{~mm}$ in antero-posterior diameter and a smaller L5-S1 protrusion (Figure 3).

The patient underwent a L4-L5 discectomy by bilateral interlamar approach, performed in the first 24 hours from presentation.

Post surgical assessment at discharge, 2 weeks post intervention, failed to demonstrate any improvement in urinary retention. She had urinary infection with Klebsiella. She followed motor rehabilitation in a specialized department.

Five weeks post surgery she was in a wheelchair after that she walked with a gait assessor.

Sensation of urine pass reappeared after 3 months. At 3 months follow-up she reported stool incontinence and sexual dysfunction with sensation preserved only in left side of vagina but with a complete block in achieving orgasm.

At 1 year follow-up she still had difficulties with bladder and bowel function and sexual problem persisted. At eight months postsurgery she start to work part time. 


\section{Discussion}

The term "cauda equina" was first described by a French anatomist, Lazarius in 1600 (7).

CES secondary to intervertebral disc herniation was first reported in 1929 by Dandy (1).

The caudal spinal cord is termed the conus medullaris with his most caudal segment situated at the level of the intervertebral disc between L1 and L2 vertebrae. Eleven pairs of nerve roots emerging from conus medullaris travels for varying distances before reaching the corresponding neural foramen, exits from spinal canal and arrives to pelvic organs (bowel, bladder), sexual organs, and also to the inferior limbs' muscles.

A common cause of cauda equina lesion is prolapse of an intervertebral disc causing nerve roots compression. In the spinal canal, the nerve roots are particularly vulnerable to injury of compressive stress because they have no Schwann cells to cover them.

Nerve root compression, especially in a stenotic canal produce a congestion and dilation of intraradicular and periradicular veins, frequently observed intraoperatively and described as varix.

In pathophysiology of CES was incriminated an autoimmune reaction (8) axonal demyelination with wallerian degeneration induced by TNF- $\alpha$ expression (9).

CES occurs when the nerve roots within the spinal canal have been damaged. The nerves supplying the muscles of the legs, the bladder, bowel and genitals do not function properly. Signs and symptoms of cauda equina root compression include leg pain and leg weakness saddle anesthesia, bladder, bowel and sexual dysfunction.
The presence of bladder or bowel dysfunction suggest the diagnosis of CES: urinary retention or overflow incontinence, constipation or faecal incontinence. Urological dysfunction and perineal sensory deficit represent the most consistent identifying features of CES. At the beginning they have difficulty in starting or stopping a stream of urine, after wards is present urinary retention or overflow incontinence.

Sexual problems are rarely reported at presentation. This is very distressing aspects, mostly in young adults, in postoperative period.

CES is a rare condition. It is generally accepted that CES occurs in approximately $1-2 \%$ of cases of lumbar disc herniations $(10,11,12,13)$.

In the management of CES patients there is considerable debate about the expediency with which surgical root decompression is carried out.

The early reports have favoured immediate decompression.

Before the mid - 1980s, authors believed that decompression should be carried out within six or eight hours after onset of acute CES.

After that, other authors stated that there is no evidence to support the statement that urgent decompression conferred great benefit when bladder dysfunction is present $(14,15,16)$.

However, the risk for poor outcome increases continuously with increasing time from onset of symptoms to decompression (17) and that patients, operated earlier did statistically better than the patients operated later (16). Determining when exactly CES is deemed to be present is difficult.

More recent studies questioned previously consensus opinion that delays in 
decompression negatively affect outcome $(18,19)$.

The influence of delays in treatment may have historically been overestimated.

Qureshi and Sell (18) consider that the major determinant of outcome may not be the timing, but the severity or density of deficit.

If surgical decompression is performed within 48 hours from onset of CES, the outcome cannot be adversely affected.

The dogma that decompression must be performed in the first 6 or 8 hours from onset is no more accepted.

If the patient with CES is operated as soon as possible after presentation in optimal conditions in the operative theater, the doctor must be absolved from any legal responsibility.

If the damage of nerve roots is incomplete, and the nerves are still capable of regrowth, recovery takes time, sometimes even several years.

In other cases, the lesion is severe and the damage should be permanent.

Our three patients had onset of symptoms since several days: back and leg pain, perineal paresthesia. They presented to hospital only when urinary dysfunction appeared. All were operated in the first 24 hours after CT-scan or MRI of lumbar spine.

However, in the postoperative period, they continue to have dysfunctions and sexual problems. One patient want to commit suicide because of sexual dysfunction.

After 1 to 4 years follow-up, with rehabilitation and medication all have a significant improvement of symptoms.

\section{Conclusion}

Cauda equina syndrome secondary to a massive lumbar disc herniation is a rare but serious condition which must be diagnosed and operated as soon as possible. Decompression performed within 48 hours from onset of symptoms absolve the surgeon from any legal responsibility and offers to the patient maximum improvement of symptoms.

\section{References}

1. Dandy WE. Loose cartilage from intervertebral disk simulating tumor of the spinal cord. Arch Surg 1929; 9: 660-672.

2. Gleave JRW, Macfarlane R. Prognosis of recovery of bladder function following lumbar central disc prolapse. Br J Neurosurg 1990; 4: 205-210.

3. Arrigo RT, Kalanithi P, BoaKyeM: Is cauda equina syndrome being treated within the recomended time frame? Neurosurgery 2011: 68: 1520-1526.

4. Shephard RH. Diagnosis and prognosis of cauda equina syndrome produced by protrusion of lumbar disk. Br Med J 1959; 2: 1434-1439.

5. Shapiro S. Cauda equina syndrome secondary to lumbar disc herniation. Neurosurg 1993; 32: 743-747.

6. Ahn UM, Ahn NU, Buchowski JM, et al. Cauda equina syndrome secondary to lumbar disc herniation: a meta-analysis of surgical outcomes. Spine 2000; 25 (12): 1515-1522.

7. Lazarius A. Historia anatomica humani corporis. Frankfurt Germany: Becker; 1600: 178.

8. Lee SK, Wolfe SW. Peripheral nerve injury and repair. J Am Acad Orthop Surg. 2000; 8(4): 243-252.

9. Sekiguchi M, Kikuchi S, Myers RR. Experimental spinal stenosis; relationship between degree of cauda equida compression, neuropathology, and pain. Spine 2004; 29 (10): 1105-1111.

10. Gitelman A, Hishmeh S, Morelli BN, et al. Cauda equina syndrome. A comprehensive review. Am J Orthop 2008; 37(11): 556-562.

11. Henriques T, Olerud C, Petre'n-Mallmin M, et al. Cauda equina syndrome as a postoperative complication in five patients operated for lumbar disc herniation. Spine 2001; 26: 293-297.

12. Jennett WB. A study of 25 cases of compression of the cauda equina by prolapsed intervertebral discs. J Neurol Neurosurg Psychiat 1956; 19: 109-116. 
13. O'Laoire SA, Crockard HA, Thomas DG. Prognosis for sphincter recovery after operation for cauda equina compression owing to lumbar disc prolapse. $\mathrm{Br}$ med J. 1981; 282: 1852-1891.

14. Kostuik JP, Harrington I, Alexander D, Rand W, Evans D. Cauda equina syndrome and lumbar disc herniation. JBone Joint Surg Arn 1986; 68: 386-391.

15. Gleave JRW, Macfarlane R. Cauda equina syndrome: what is the relationship between timing of surgery and outcome? Br J Neurosurg 2002; 16 (4): 325-328.

16. Shapiro S. Medical realities of cauda equina syndrome secondary to lumbar disc herniation. Spine 2000; 25: 348-352.

17. Kohles DA, Karp AP, et al. Time-depended surgical outcomes following cauda equina syndrome diagnosis:comments on a meta-analysis. Spine. 2004; 29(11): 1281-1287.

18. Qureshi A, Sell P: Cauda equina syndrome treated by surgical decompression: the influence of timing on surgical outcome. Eur Spine. 2007; 16(12): 2143-2151. 19. Hussain SA, Gallan RW, Chitnavis BP. Cauda equina syndrome outcome and implications for management. Br J Neurosurg. 2003; 17(2): 164-167. 